\title{
Lambda-Cyhalothrin Nanosuspension Prepared by the Melt Emulsification-High Pressure Homogenization Method
}

\author{
Zhenzhong Pan, ${ }^{1,2}$ Bo Cui, ${ }^{1}$ Zhanghua Zeng, ${ }^{1}$ Lei Feng, ${ }^{1}$ Guoqiang Liu, ${ }^{1}$ \\ Haixin Cui, ${ }^{1}$ and Hongyu Pan $^{2}$ \\ ${ }^{1}$ Institute of Environment and Sustainable Development in Agriculture, Chinese Academy of Agricultural Sciences, \\ Beijing 100081, China \\ ${ }^{2}$ College of Natural Resources and Environment, College of Plant Science, Jilin University, Changchun 130062, China \\ Correspondence should be addressed to Haixin Cui; haixincui@caas.cn and Hongyu Pan; panhongyu@jlu.edu.cn
}

Received 22 April 2015; Revised 3 July 2015; Accepted 8 July 2015

Academic Editor: Jin-Ho Choy

Copyright (C) 2015 Zhenzhong Pan et al. This is an open access article distributed under the Creative Commons Attribution License, which permits unrestricted use, distribution, and reproduction in any medium, provided the original work is properly cited.

\begin{abstract}
The nanosuspension of $5 \%$ lambda-cyhalothrin with $0.2 \%$ surfactants was prepared by the melt emulsification-high pressure homogenization method. The surfactants composition, content, and homogenization process were optimized. The anionic surfactant (1-dodecanesulfonic acid sodium salt) and polymeric surfactant (maleic rosin-polyoxypropylene-polyoxyethylene ether sulfonate) screened from 12 types of commercially common-used surfactants were used to prepare lambda-cyhalothrin nanosuspension with high dispersity and stability. The mean particle size and polydispersity index of the nanosuspension were $16.01 \pm 0.11 \mathrm{~nm}$ and $0.266 \pm 0.002$, respectively. The high zeta potential value of $-41.7 \pm 1.3 \mathrm{mV}$ and stable crystalline state of the nanoparticles indicated the excellent physical and chemical stability. The method could be widely used for preparing nanosuspension of various pesticides with melting points below boiling point of water. This formulation may avoid the use of organic solvents and reduce surfactants and is perspective for improving bioavailability and reducing residual pollution of pesticide in agricultural products and environment.
\end{abstract}

\section{Introduction}

Pesticides have been widely applied in integrated pest management of crop production. However, most of effective pesticide compounds have poor water solubility, which limits the further development of water-based formulations $[1,2]$. There are some problems on conventional pesticide formulations such as emulsifiable concentrate (EC), wettable powders (WP), and microemulsion (ME), including the use of organic solvent, drift, and rainfastness, which caused low efficacy and severely environmental contamination [3]. Nanosuspension has been considered to be an important and promising candidate to overcome the problems mentioned above due to the significant increase on aqueous dispersity and solubility of nanoparticle as described by Ostwald-Freundlich equation [4-7].

Pesticide nanosuspension is nanocolloidal dispersion of pesticide active compound particles in crystal or amorphous state stabilized by surfactants $[8,9]$. Nanosuspension not only could solve the problem of poor solubility but also could improve permeability, bioactivity, and efficacy of pesticide [10]. Generally, nanosuspension in pharmaceutical field can be produced either by top-down approaches, such as media milling, high pressure homogenization ( $\mathrm{HPH})$, melt emulsification method, and high pressure microfluidization, or bottom-up techniques, such as precipitation and supercritical fluid method [11-13]. Recently, the combination of the melt emulsification and HPH method (melt emulsification-HPH method) [14] has attracted intense attentions, owing to avoiding use of organic solvents and reducing the energy consumption of milling during the production process. However, the related research in pesticide formulations was hardly reported. The basic processes of the melt emulsificationHPH method are described as follows. The pesticide was melted in water at temperature above the melting point and emulsified in high pressure homogenizer. The effectiveness of HPH is related to the hardness of the drug particle, the homogenization pressure, and the number of cycles [15-18]. 


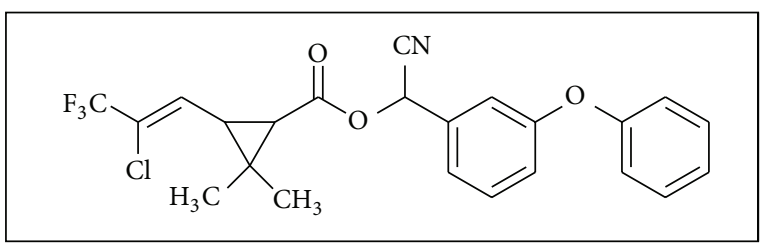

FIGURE 1: Chemical structure of lambda-cyhalothrin.

As shown in Figure 1, lambda-cyhalothrin is a very poor water soluble synthetic pyrethroid insecticide $\left(5 \times 10^{-6} \mathrm{~g} / \mathrm{L}\right.$ at $25^{\circ} \mathrm{C}$ ) and highly effective against a wide range of pests [19]. The current formulations of lambda-cyhalothrin include WP, EC, ME, and emulsion in water (EW) [20, 21].

In this study, we prepared lambda-cyhalothrin nanosuspension by the melt emulsification-HPH method with the combination of anionic and polymeric surfactants. The particle size and distribution of the nanosuspension were investigated by dynamic light scattering (DLS). The morphology and stability of particle were characterized by scanning electron microscopy (SEM), X-ray diffraction (XRD), and high performance liquid chromatography (HPLC).

\section{Experimental Methods}

2.1. Materials. Lambda-cyhalothrin (96\%) was purchased from Yangnong Chemical Co., Ltd. Polycarboxylate and maleic rosin-polyoxypropylene-polyoxyethylene ether sulfonate (MRES) were provided by Jiangsu Sinvochem S\&D., Ltd. 1-Dodecanesulfonic acid sodium salt (SDS), sodium lauryl ether sulfate (SLES), polyvinylpyrrolidone K30 (PVP K30), polyvinylpyrrolidone K90 (PVP K90), sodium lignin sulfonate (SL), and polyoxyethylene sorbitan monooleate (Tween 80) were purchased from J\&K Chemical. Polyethylene glycol mono-4-nonylphenyl ether (PEGNPE) was purchased from Alfa Aesar. Hydroxypropyl methyl cellulose (HPMC), poloxamer 188 (F68), sorbitan oleate (Span 80), and mannitol were obtained from Sigma-Aldrich. HPLC grade methanol was purchased from Fisher. The water used in all analytical experiments was Milli-Q water $(18.2 \mathrm{M} \Omega \cdot \mathrm{cm}$, $\mathrm{TOC} \leq 4 \mathrm{ppb})$.

\subsection{Methods}

2.2.1. The Preparation of Lambda-Cyhalothrin Nanosuspension. Lambda-cyhalothrin nanosuspension was produced by the melt emulsification-HPH method. Lambda-cyhalothrin powder was dispersed in deionized water containing surfactants at $80^{\circ} \mathrm{C}$ (above the melting point of lambdacyhalothrin) and emulsified with C25 shearing machine (ATS, Germany) at 10,000 rpm for $2 \mathrm{~min}$. Subsequently, the emulsified mixture was homogenized using AH100D high pressure homogenizer (ATS Engineer Inc., Canada) under different pressures in the range of 200-1000 bar for 15 cycles. Finally, the nanosuspension was obtained by cooling down the homogenized mixture to room temperature in order to solidify the melted droplets into nanoparticles. The total procedures of the flow were shown in Figure 2.
2.2.2. Particle Size and Zeta Potential of the Nanosuspension. The mean particle size, $90 \%$ of the particle volume below a certain size (D90), polydispersity index (PDI), and zeta potential of the nanosuspension were measured by DLS with a Zetasizer Nano ZS90 (Malvern Instruments, UK) at $25^{\circ} \mathrm{C}$ at a scattering angle of $90^{\circ}$. The measurement of each sample was carried out in triplicate for reliability.

2.2.3. Morphology Characterization of Nanoparticles. The morphology of lambda-cyhalothrin nanoparticles was investigated by JSM-7401F scanning electron microscopy (SEM) (JEOL, Japan) with an accelerating voltage of $3 \mathrm{kV}$. The samples were dropped on silicon slice, dried naturally, and coated with a thin layer of platinum for $30 \mathrm{~s}$ using ETD-800 sputter coater (Beijing Elaborate Technology Development Ltd., China).

2.2.4. Crystalline State Analysis of Nanoparticles. The solid powder of lambda-cyhalothrin nanoparticles was prepared from the nanosuspension by freeze drying using FD-81 freeze dryer (EYELA, Japan) for $24 \mathrm{~h}$ at $0.4 \mathrm{~Pa}$. The crystalline state was characterized by D8 Advance X-ray diffractometer (Bruker AXS Inc., Germany) with a $\mathrm{Cu} \mathrm{K} \alpha$ radiation generated at $40 \mathrm{kV}$ and $40 \mathrm{~mA}$ current. Samples were analyzed in a $2 \theta$ range of $8-55^{\circ}$, with a step size of $0.02^{\circ}$ and a time step of $0.1 \mathrm{~s}$.

2.2.5. Stability Measurement. The suspensibility test was conducted according to GB/T 14825-2006, China [22]. $5.0 \mathrm{~g}$ lambda-cyhalothrin nanosuspension was diluted in $250 \mathrm{~mL}$ standard hard water at $30^{\circ} \mathrm{C}$. The diluted nanosuspension was placed under $30^{\circ} \mathrm{C}$ water bath for $30 \mathrm{~min}$. Then the top $225 \mathrm{~mL}$ of the solution was removed. The drug contents of the original suspension and the left $25 \mathrm{~mL}$ solution were measured by HPLC. The suspensibility $(w)$ was calculated by the following equation:

$$
w(\%)=\frac{m_{1}-m_{2}}{m_{1}} \times \frac{10}{9} \times 100,
$$

where $m_{1}$ and $m_{2}$ are the pesticide content of original suspension and the left $25 \mathrm{~mL}$ solution at the bottom, respectively. The unit for $m_{1}$ and $m_{2}$ is gram (g).

The storage stability was tested according to HG/T 2467.52003, China [23]. The nanosuspension was stored in a closed glass vial at $0^{\circ} \mathrm{C}$ for 7 days and $54^{\circ} \mathrm{C}$ for 14 days. After storage, samples were drawn to assess physical and chemical stability. Chemical stability was tested by analyzing lambdacyhalothrin remaining of the nanosuspension with HPLC.

2.2.6. HPLC Analysis. Lambda-cyhalothrin content was analyzed by HPLC (Waters Alliance 2695) at room temperature using WATO45905 C18 analytical column and $278 \mathrm{~nm} \mathrm{UV}$ detector. The mobile phase was a mixture of methanol and water $(80: 20, \mathrm{v} / \mathrm{v})$ and the flow rate was $1.0 \mathrm{~mL} / \mathrm{min}$.

2.2.7. Statistical Analysis. All measurements were conducted in triplicate. The data were recorded as mean \pm standard deviation and analyzed by one-way analysis of variance 


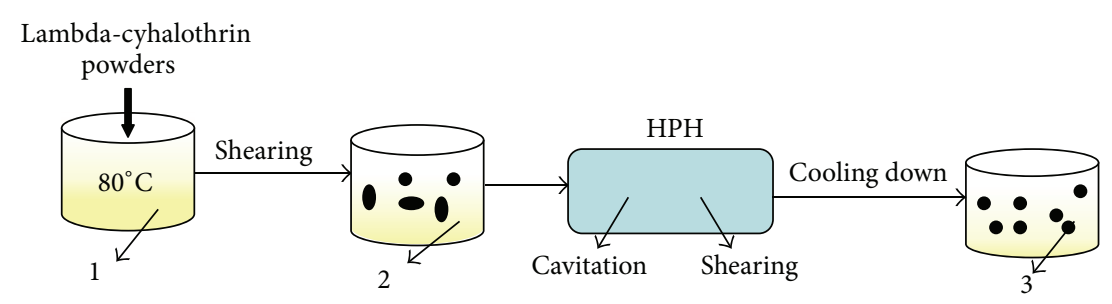

FIGURE 2: Schematic representation of lambda-cyhalothrin nanosuspension preparation. (1) Surfactant aqueous dispersion. (2) The emulsified mixture. (3) Lambda-cyhalothrin nanosuspension.

TABLE 1: The influence of surfactant on the mean particle size and PDI of lambda-cyhalothrin nanosuspension.

\begin{tabular}{lcc}
\hline Surfactant & Mean particle size $(\mathrm{nm})$ & PDI \\
\hline SL & $210.13 \pm 2.40 \mathrm{e}$ & $0.137 \pm 0.006 \mathrm{c}$ \\
SDS & $19.95 \pm 0.04 \mathrm{~h}$ & $0.389 \pm 0.009 \mathrm{ab}$ \\
F68 & $201.53 \pm 2.50 \mathrm{e}$ & $0.231 \pm 0.023 \mathrm{bc}$ \\
SLES & $207.73 \pm 1.86 \mathrm{e}$ & $0.169 \pm 0.016 \mathrm{bc}$ \\
MRES & $157.63 \pm 1.02 \mathrm{fg}$ & $0.233 \pm 0.024 \mathrm{bc}$ \\
HPMC & $517.07 \pm 19.20 \mathrm{a}$ & $0.496 \pm 0.398 \mathrm{a}$ \\
PEGNPE & $149.60 \pm 2.25 \mathrm{~g}$ & $0.172 \pm 0.028 \mathrm{bc}$ \\
Tween 80 & $165.30 \pm 3.24 \mathrm{f}$ & $0.202 \pm 0.005 \mathrm{bc}$ \\
Span 80 & $239.20 \pm 5.73 \mathrm{~d}$ & $0.238 \pm 0.028 \mathrm{bc}$ \\
PVP K30 & $335.84 \pm 3.98 \mathrm{~b}$ & $0.130 \pm 0.014 \mathrm{c}$ \\
PVP K90 & $279.13 \pm 2.64 \mathrm{c}$ & $0.155 \pm 0.052 \mathrm{bc}$ \\
Polycarboxylate & $247.97 \pm 1.80 \mathrm{~d}$ & $0.139 \pm 0.026 \mathrm{c}$ \\
\hline
\end{tabular}

Different letters at each data indicate significant differences according to Duncan's multiple range test at $P<0.05$.

(ANOVA) and Duncan's multiple range test. Significance was tested at the 0.05 level of probability. Statistical analysis was performed with the software package SPSS.

\section{Results and Discussion}

3.1. The Influence of Surfactants on the Particle Size and Dispersity of Lambda-Cyhalothrin Nanosuspension. When the surfactant content was less than half the active pesticide ingredient, rapid deposition phenomenon was observed in most suspensions stabilized by single surfactant, so the $2: 1$ ratio of lambda-cyhalothrin to surfactant was determined to screen the optimal surfactants for lambda-cyhalothrin nanosuspension. The nanosuspension containing $1 \%(w / w)$ lambda-cyhalothrin and $0.5 \%$ surfactant $(w / w)$ was prepared by the melt emulsification-HPH method. The statistical result revealed that surfactant had significant impact on the particle size and dispersity of the nanosuspension $(P<0.05)$. As shown in Table 1, among twelve surfactants, four surfactants (SDS, MRES, Tween 80, and PEGNPE) reduced the mean particle size of the nanosuspension less than $200 \mathrm{~nm}$; in particular the SDS reduced mean particle size to as low as $19.95 \pm 0.04 \mathrm{~nm}$. As anionic surfactant, SDS can keep the
TABle 2: The influence of complex surfactants on the particle size and dispersity of the nanosuspension.

\begin{tabular}{lccc}
\hline Formulation $^{\mathrm{a}}$ & $\begin{array}{c}\text { Mean particle } \\
\text { size }(\mathrm{nm})\end{array}$ & PDI & D90 \\
\hline NS-1 & $16.01 \pm 0.11 \mathrm{c}$ & $0.266 \pm 0.002 \mathrm{a}$ & $54.00 \pm 11.00 \mathrm{~b}$ \\
NS-2 & $40.45 \pm 0.27 \mathrm{~b}$ & $0.275 \pm 0.008 \mathrm{a}$ & $119.03 \pm 27.00 \mathrm{a}$ \\
NS-3 & $44.44 \pm 0.96 \mathrm{a}$ & $0.235 \pm 0.017 \mathrm{~b}$ & $120.67 \pm 7.77 \mathrm{a}$ \\
\hline
\end{tabular}

Note: ${ }^{\mathrm{a} N S}-1:$ SDS + MRES $(1: 3, \mathrm{w} / \mathrm{w})$, NS-2: SDS + PEGNPE $(1: 3, \mathrm{w} / \mathrm{w})$, and NS-3: SDS + Tween $80(1: 3, \mathrm{w} / \mathrm{w})$.

Different letters at each data indicate significant differences according to Duncan's multiple range test at $P<0.05$.

pesticide particles well dispersed via electrostatic repulsion [24]. MRES is anionic polymer which can afford electrostatic repulsion and substantially steric barrier at the interface of individual particle [25]. Nonionic surfactants, PEGNPE and Tween 80, could inhibit aggregation of particles by adsorbing onto the nanoparticles through the hydrophobic section. It seems that interactions between hydrophobic areas of lambda-cyhalothrin, PEGNPE, and Tween 80 were substantial for nanoparticles [26]. Therefore, SDS, PEGNPE, Tween 80, and MRES were chosen for further optimization.

Although SDS decreased significantly the particle size of the nanosuspension, the high PDI value indicated the poor dispersity in water. Based on the result of surfactant screening, the complex effect of surfactants was considered and further investigated. It has been reported that the combination of anionic surfactant and nonionic surfactant could offer electrostatic and steric repulsion for the nanosuspension stabilization $[27,28]$. Therefore, the complex effect of SDS with PEGNPE, Tween 80, and MRES was investigated on the particle size and dispersity of the nanosuspension with $5 \%(w / w)$ lambda-cyhalothrin and $0.2 \%(w / w)$ surfactants. Table 2 presented the measuring results of the particle size and dispersity of three nanosuspension formulations with complex surfactants, NS-1 (containing SDS and MRES), NS2 (containing SDS and PEGNPE), and NS-3 (containing SDS and Tween 80). Among the three formulations, smallest particle size and narrow distribution were observed with the complex surfactants of SDS and MRES $(1: 3, w / w)$ in NS-1, which was significantly smaller than those of the other two nanosuspensions $(P<0.05)$. Thus, SDS and MRES $(1: 3$, $w / w)$ were chosen as the optimal complex surfactants. 


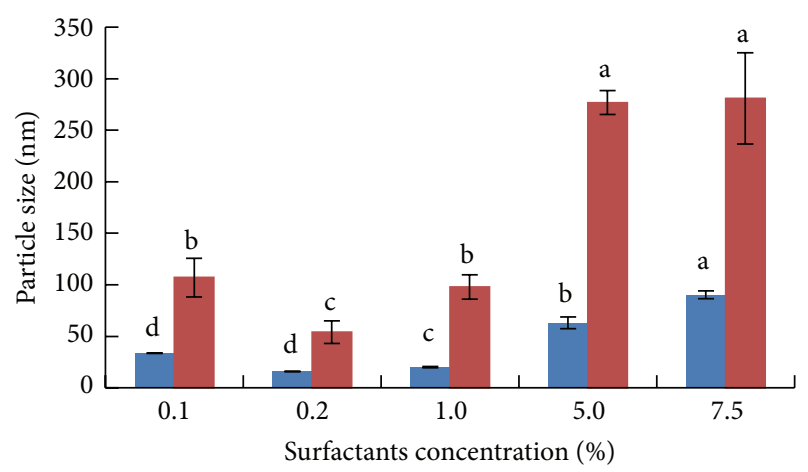

Mean particle size D 90

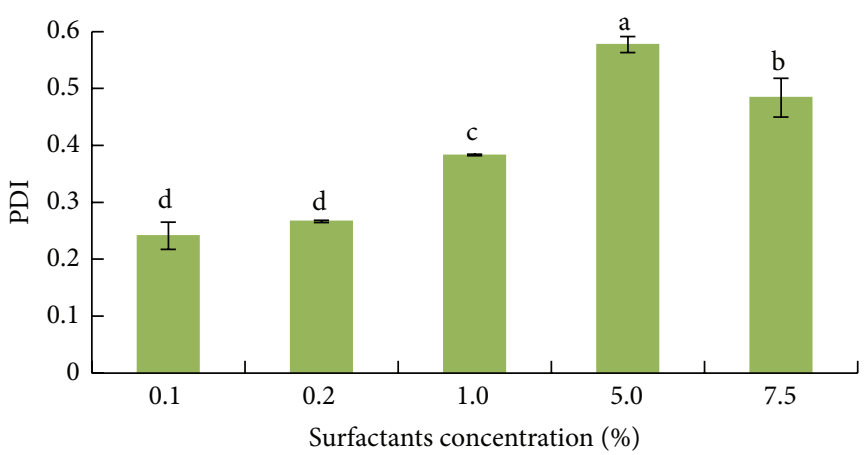

PDI

(a)

(b)

FIGURE 3: Effect of surfactants concentration on (a) particle size and (b) PDI of the nanosuspension. Different letters at each data indicate significant differences according to Duncan's multiple range test at $P<0.05$.

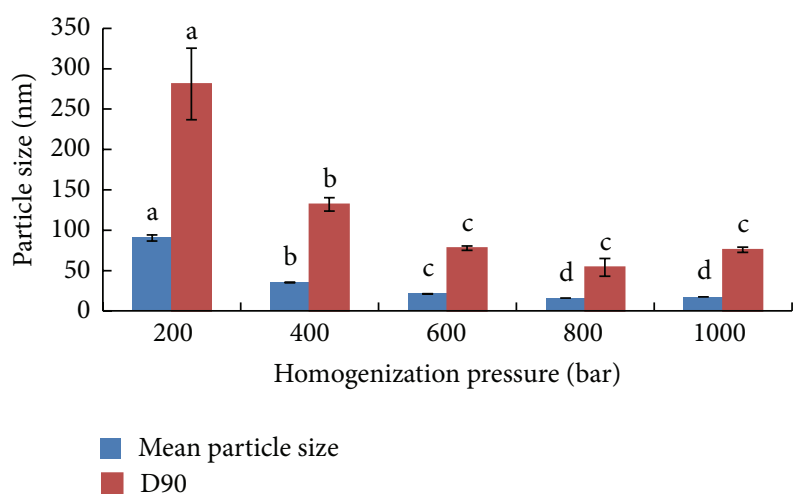

(a)

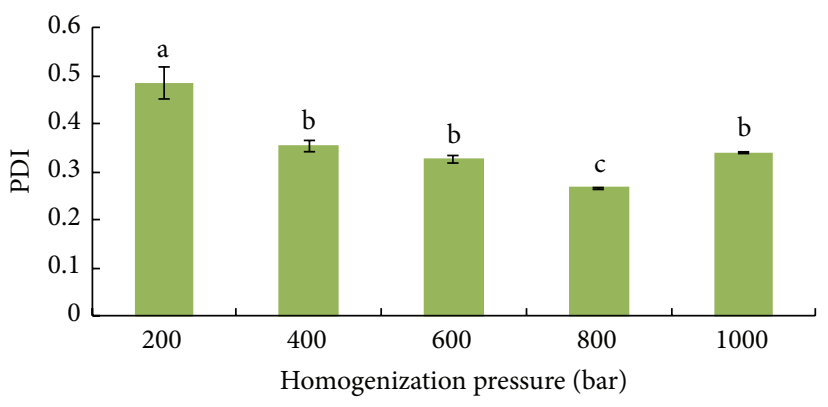

PDI

FIGURE 4: Effect of homogenization pressure on (a) particle size and (b) PDI of the nanosuspension. Different letters at each data indicate significant differences according to Duncan's multiple range test at $P<0.05$.

3.2. Optimization of the Surfactants Concentration. As shown in Figure 3, mean particle size and D90 of lambda-cyhalothrin nanosuspension were reduced along with increase on the concentration of surfactants from $0.1 \%$ to $0.2 \%(w / w)$ and increased significantly from $0.2 \%$ to $7.5 \%(w / w)(P<0.05)$. Presumably, at low concentration, the surfactant reduced the interfacial tension and prevented drug particles from aggregating; when both complex surfactants' concentration reached a critical micelle concentration $\left(\mathrm{CMC}_{\mathrm{MRES}}=1.30 \mathrm{~g} / \mathrm{L}\right.$ and $\mathrm{CMC}_{\mathrm{SDS}}=2.45 \mathrm{~g} / \mathrm{L}$ at $25^{\circ} \mathrm{C}$ ), the drug particles were wrapped in the micelles, giving rise to increase on particle size. Additionally, PDI exhibited similar trend to particle size, while the surfactants concentration changed from $0.2 \%$ to $7.5 \%(w / w)$. Therefore, $0.2 \%(w / w)$, a proper concentration of surfactant, was chosen for the preparation of lambdacyhalothrin nanosuspension.

3.3. Optimization of the Homogenization Condition. Generally, the homogenization pressure is the key parameter of $\mathrm{HPH}$ process to determine the particle size of the nanosuspension [29] and the effect has been proved in this investigation
$(P<0.05)$. Thus, the cycles were fixed at 15 for each pressure and the various $\mathrm{HPH}$ pressures were investigated in detail. As shown in Figure 4, the mean particle size, D90, and PDI decreased as pressure increased from 200 to 800 bar. At 800 bar, the mean particle size and D90 were $16.01 \pm 0.11 \mathrm{~nm}$ and $54.00 \pm 11.00 \mathrm{~nm}$ at 800 bar, respectively. When the pressure was above 800 bar, D90 and PDI significantly increased along with slight increase on mean particle size. It seemed that continuous increase on pressure could not afford reduction of particle size.

Therefore, the optimal formulation was the fact that $5 \%(w / w)$ lambda-cyhalothrin nanosuspension with $0.2 \%$ surfactants $(w / w)$ of SDS and MRES $(1: 3, w / w)$ was prepared by the melt emulsification-HPH method of 800 bar with 15 cycles.

3.4. Morphology of the Nanosuspension. The particles of lambda-cyhalothrin nanosuspension presented sphere-like shape as observed in SEM imaging (Figure 5(a)). The statistical mean particle size based on 100 particles from SEM imaging was $57.07 \mathrm{~nm}$ (Figure 5(b)), which was well in 


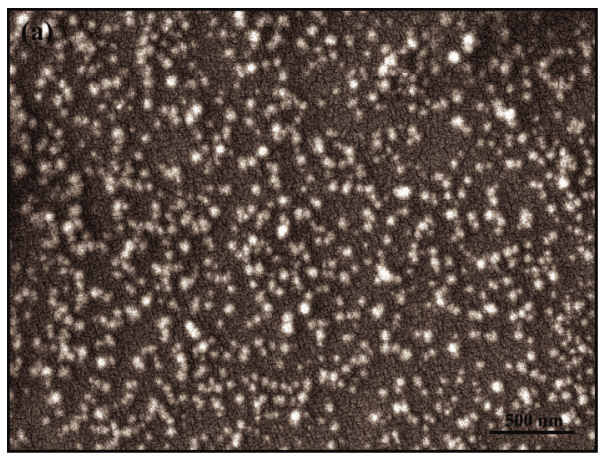

(a)

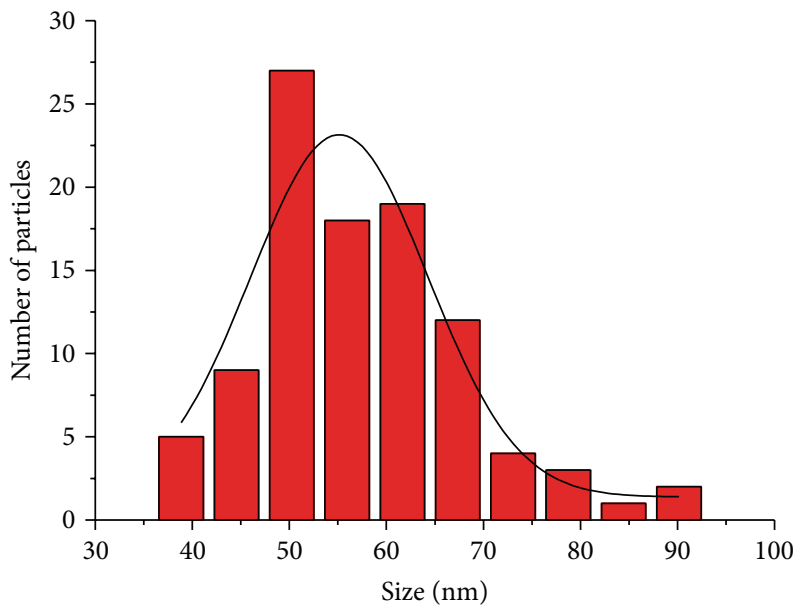

(b)

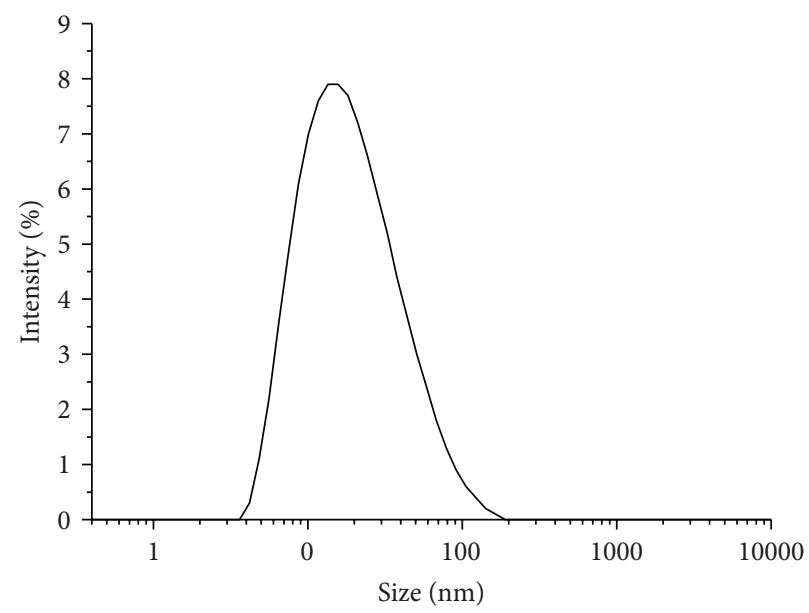

(c)

FIGURE 5: (a) SEM imaging, (b) statistical particle size based on SEM image (100 particles), and (c) hydrodynamic particle size distribution of lambda-cyhalothrin nanosuspension measured by DLS.

agreement with the D90 measured by DLS (Figure 5(c)). This could be attributed to the shear and cavitation effect, disintegrating large particles into nanosized particles during the HPH process [30].

3.5. Characterization of Crystalline State. It is well known that the crystalline state of nanoparticles significantly influences the solubility, stability, and bioavailability of nanosuspension formulations [31]. High pressure adopted in this process may change the crystalline state of lambda-cyhalothrin [32]. Therefore, the effect on the crystalline state was exploited with freeze-drying method which has a good ability to preserve the original structure of materials combined with surfactants during drying $[33,34]$. Figure 6 showed the X-ray powder diffractogram profiles of MRES, SDS, mannitol, and lambda-cyhalothrin, physical mixture of them, and lambdacyhalothrin nanoparticles. The typical diffraction peaks of lambda-cyhalothrin remained in the nanoparticles after the $\mathrm{HPH}$ and freeze-drying, indicating the crystalline state of the lambda-cyhalothrin kept almost unchanged. The crystalline state of lambda-cyhalothrin nanoparticles is beneficial to long-term stability [31]. Meanwhile, the crystallite size of lambda-cyhalothrin nanoparticle was calculated to be $57.30 \mathrm{~nm}$ with Scherrer's equation, which was similar to the particle size measured by DLS and SEM. This result revealed the good stability during solidification and dispersity of the pesticide nanoparticles in solution.

3.6. Stability of Nanosuspension. As shown in Figure 7, the suspensibility of the nanosuspension was $99.1 \pm 0.3 \%$ initially. After storage at $0^{\circ} \mathrm{C}$ for 7 days and $54^{\circ} \mathrm{C}$ for 14 days, the suspensibilities of the nanosuspension were $98.2 \pm 0.3 \%$ and $97.7 \pm 0.4 \%$, respectively, indicating its high stability. In addition, the zeta potential of lambda-cyhalothrin nanosuspension remained $-41.7 \pm 1.3 \mathrm{mV}$ owing to the coating of SDS and MRES. It is well known that absolute value of zeta potential of approximate $30 \mathrm{mV}$ exhibits enough electrostatic repulsion between the nanoparticles to prohibit aggregation [29]. Furthermore, the anionic polymer MRES on the surface of particle offers steric hindrance to enhance stability. As shown in Figure 8, the mean particle size of the nanosuspension was $16.01 \pm 0.11 \mathrm{~nm}$ initially and basically 


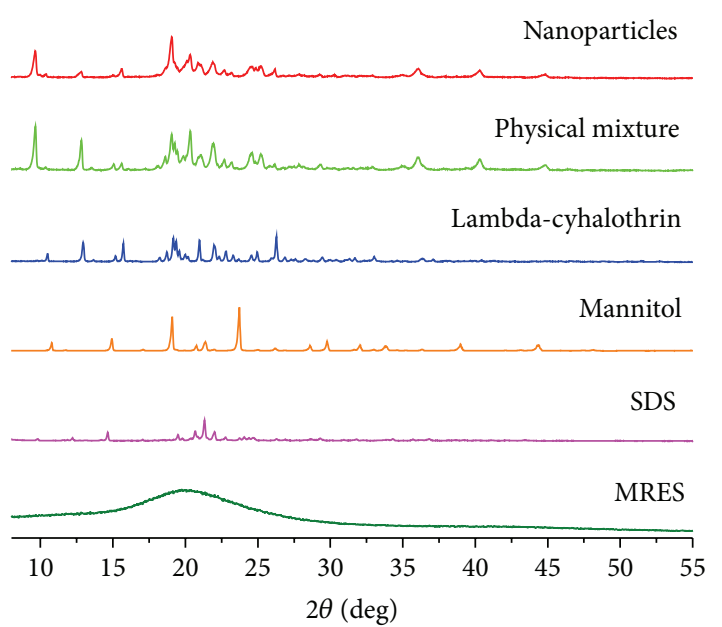

FIGURE 6: X-ray diffraction patterns of lambda-cyhalothrin and related excipients.

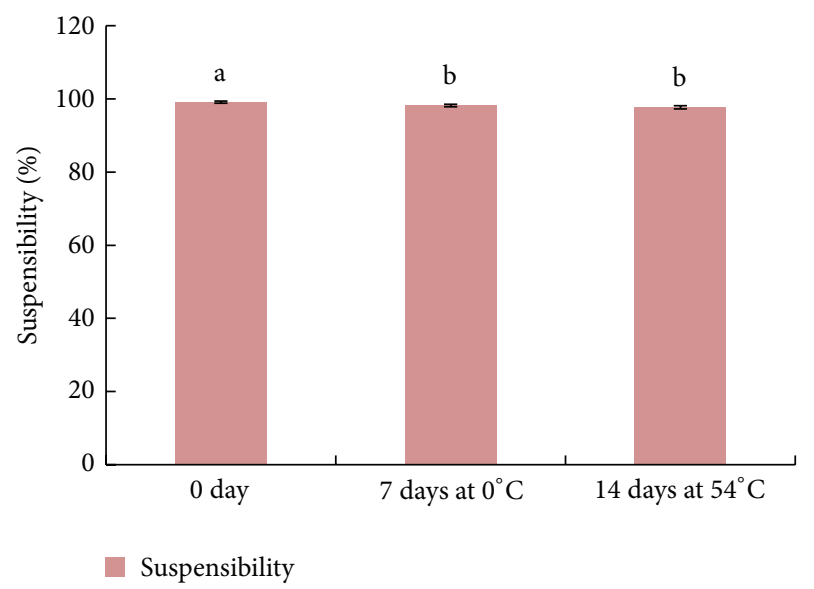

Figure 7: The suspensibility of the nanosuspension under different storage conditions. Different letters at each data indicate significant differences according to Duncan's multiple range test at $P<0.05$.

remained unchanged after storage at $0^{\circ} \mathrm{C}$ for 7 days. Meanwhile, at $54^{\circ} \mathrm{C}$ for 14 days, the mean particle size increased to $24.95 \pm 0.65 \mathrm{~nm}$, an increase caused by aggregation of fewer particles. On the other hand, PDI of the nanosuspension decreased slightly, indicating excellent stability. These results suggested sufficient physical stability of lambda-cyhalothrin nanosuspension for storage.

In addition, the lambda-cyhalothrin concentration kept almost constant at $0^{\circ} \mathrm{C}$ for 7 days and $54^{\circ} \mathrm{C}$ for 14 days indicating that the nanosuspension showed an excellent chemical stability (Figure 9). Presumably, the surrounding surfactants protected lambda-cyhalothrin molecules from degradation. As shown in Figure 10, there is no apparent difference in the XRD powder diffractograms, indicating that the crystalline state of lambda-cyhalothrin nanoparticles was stable. Overall, the nanosuspension of lambda-cyhalothrin prepared by the melt emulsification-HPH method presented excellent stability in physical and chemical properties.

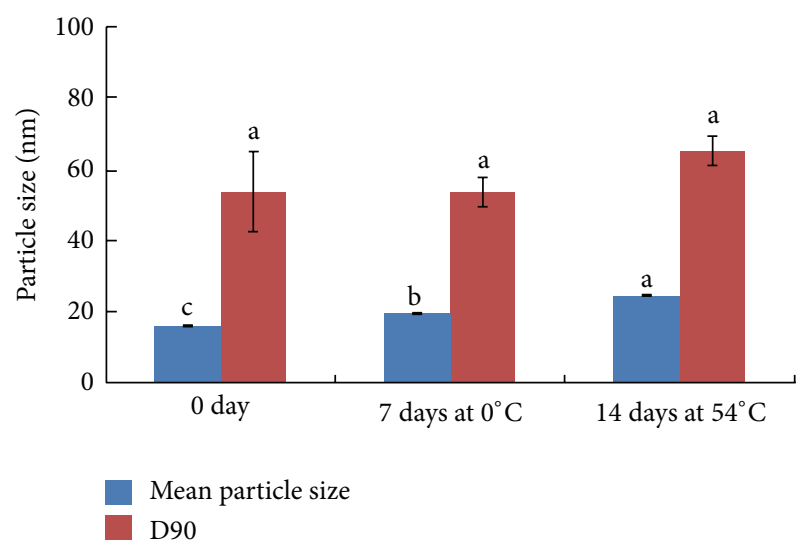

(a)

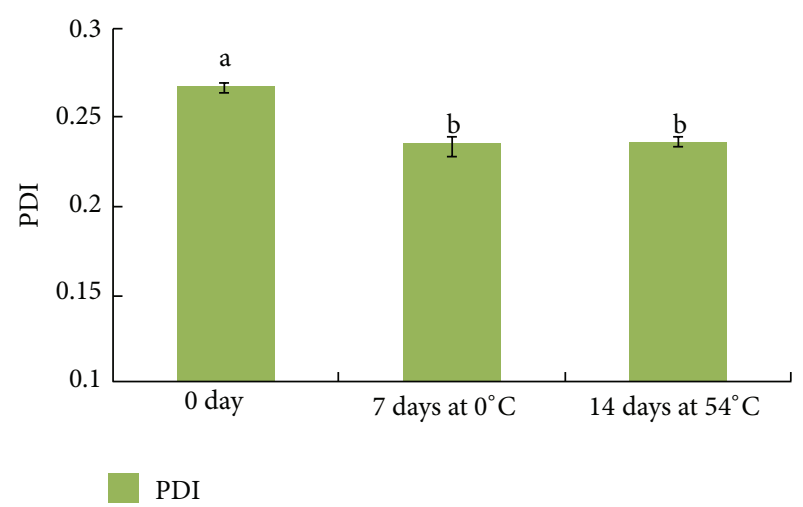

(b)

FIgURE 8: The changes of (a) particle size and (b) PDI of the nanosuspension under different storage conditions. Different letters at each data indicate significant differences according to Duncan's multiple range test at $P<0.05$.

\section{Conclusion}

In summary, the nanosuspension of $5 \%(w / w)$ lambdacyhalothrin containing $0.2 \%$ surfactants $(w / w)$ was prepared with high dispersity and stability by the melt emulsification$\mathrm{HPH}$ method. It was demonstrated that the surfactants and homogenization pressure had significant effects on particle size and dispersity of the nanosuspension. The anionic surfactant (SDS) and polymeric surfactant (MRES) screened from 12 types of common-used surfactants were determined to be optimal complex surfactant for the preparation of nanosuspension. The nanosuspension was less than $100 \mathrm{~nm}$ in particle size and dispersed uniformly with high zeta potential value and excellent stability.

The nanosuspension produced by the melt emulsification- $\mathrm{HPH}$ avoids the use of organic solvents and reduces the use of surfactants compared with EC and ME and could be applicable to various pesticides with melting points below boiling point of water. Therefore, this kind of formulation is perspective in plant protection for improving bioavailability and reducing residual pollution of pesticide in agricultural products and environment. 


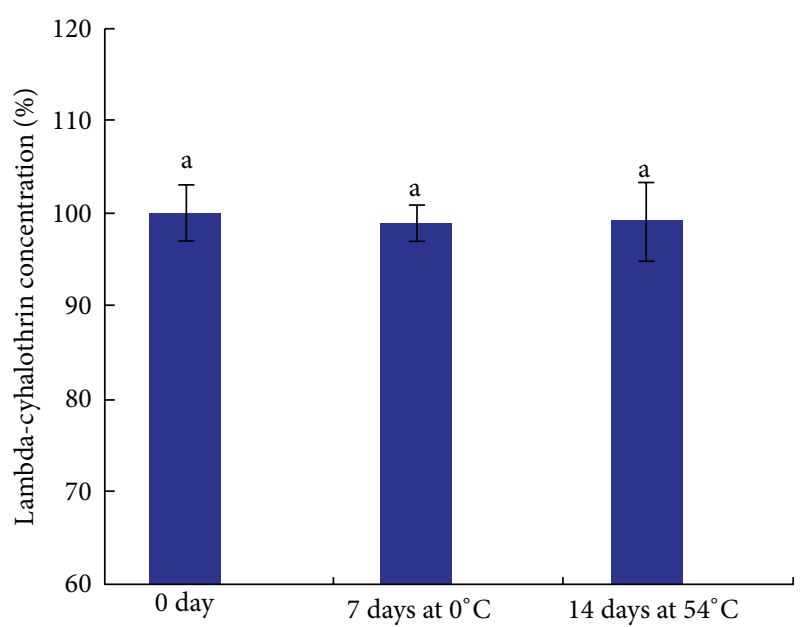

FIGURE 9: The chemical stability of the nanosuspension under different storage conditions. Same letter at each data indicates that there is no significant difference according to Duncan's multiple range test at $P<0.05$.
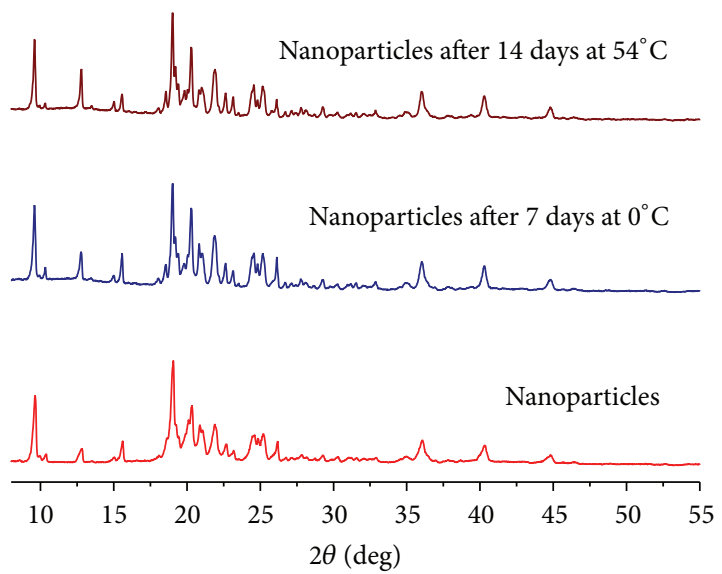

FIGURE 10: X-ray diffraction patterns of lambda-cyhalothrin nanosuspension after storage.

\section{Conflict of Interests}

The authors declare that there is no conflict of interests regarding the publication of this paper.

\section{Authors' Contribution}

Zhenzhong Pan and Bo Cui contributed equally to this work.

\section{Acknowledgments}

The research was supported by Major National Scientific Research Program of China (no. 2014CB932200), Basic Scientific Research Foundation of National Non-Profit Scientific Institute of China (no. BSRF201406), National 863 program of the People's Republic of China (no. 2011AA10A201), the 12th Five-Year Plan Project of Science and Technology Support of China (2014BAD14B02 and 2013BAC09B01), and
Research and Development of Industrial Technology Special at Jilin Provincial Development and Reform Commission (2013C001).

\section{References}

[1] Y. Ci, Y. J. Wen, X. L. Han et al., "Research progress of nano-TiO botanical pesticide," Chinese Journal of Hygienic Insecticides and Equipments, vol. 19, no. 1, pp. 73-79, 2013.

[2] A. Goswami, I. Roy, S. Sengupta, and N. Debnath, "Novel applications of solid and liquid formulations of nanoparticles against insect pests and pathogens," Thin Solid Films, vol. 519, no. 3, pp. 1252-1257, 2010.

[3] Z. Z. Pan, C. Cui, H. X. Cui et al., "Progress on pesticide nanosuspension and its preparation methods," Chinese Journal of Pesticide Science, vol. 16, no. 6, pp. 635-643, 2014.

[4] S. M. D’Addio and R. K. Prud'homme, "Controlling drug nanoparticle formation by rapid precipitation," Advanced Drug Delivery Reviews, vol. 63, no. 6, pp. 417-426, 2011.

[5] V. Ghormade, M. V. Deshpande, and K. M. Paknikar, "Perspectives for nano-biotechnology enabled protection and nutrition of plants," Biotechnology Advances, vol. 29, no. 6, pp. 792-803, 2011.

[6] C. H. Anjali, S. Sudheer Khan, K. Margulis-Goshen, S. Magdassi, A. Mukherjee, and N. Chandrasekaran, "Formulation of water-dispersible nanopermethrin for larvicidal applications," Ecotoxicology and Environmental Safety, vol. 73, no. 8, pp. 19321936, 2010.

[7] N. Elek, R. Hoffman, U. Raviv, R. Resh, I. Ishaaya, and S. Magdassi, "Novaluron nanoparticles: formation and potential use in controlling agricultural insect pests," Colloids and Surfaces A: Physicochemical and Engineering Aspects, vol. 372, no. 1-3, pp. 66-72, 2010.

[8] R. S. Suresh Kumar, P. J. Shiny, C. H. Anjali et al., "Distinctive effects of nano-sized permethrin in the environment," Environmental Science and Pollution Research, vol. 20, no. 4, pp. 25932602, 2013.

[9] H. Zeng, X. F. Li, G. Zhang, and J. Dong, "Preparation and characterization of beta cypermethrin nanosuspensions by diluting O/W microemulsions," Journal of Dispersion Science and Technology, vol. 29, no. 3, pp. 358-361, 2008.

[10] K. Kawakami, "Modification of physicochemical characteristics of active pharmaceutical ingredients and application of supersaturatable dosage forms for improving bioavailability of poorly absorbed drugs," Advanced Drug Delivery Reviews, vol. 64, no. 6, pp. 480-495, 2012.

[11] J. P. Möschwitzer, "Drug nanocrystals in the commercial pharmaceutical development process," International Journal of Pharmaceutics, vol. 453, no. 1, pp. 142-156, 2013.

[12] B. Sun and Y. Yeo, "Nanocrystals for the parenteral delivery of poorly water-soluble drugs," Current Opinion in Solid State and Materials Science, vol. 16, no. 6, pp. 295-301, 2012.

[13] L. B. Wu, J. Zhang, and W. Watanabe, "Physical and chemical stability of drug nanoparticles," Advanced Drug Delivery Reviews, vol. 63, no. 6, pp. 456-469, 2011.

[14] P. Kocbek, S. Baumgartner, and J. Kristl, "Preparation and evaluation of nanosuspensions for enhancing the dissolution of poorly soluble drugs," International Journal of Pharmaceutics, vol. 312, no. 1-2, pp. 179-186, 2006.

[15] C. M. Keck and R. H. Müller, "Drug nanocrystals of poorly soluble drugs produced by high pressure homogenisation," 
European Journal of Pharmaceutics and Biopharmaceutics, vol. 62, no. 1, pp. 3-16, 2006.

[16] M. Talekar, S. Ganta, M. Amiji et al., "Development of PIK-75 nanosuspension formulation with enhanced delivery efficiency and cytotoxicity for targeted anti-cancer therapy," International Journal of Pharmaceutics, vol. 450, no. 1-2, pp. 278-289, 2013.

[17] Y. C. Wang, Y. Y. Ma, Y. Zheng et al., "In vitro and in vivo anticancer activity of a novel puerarin nanosuspension against colon cancer, with high efficacy and low toxicity," Drug Development and Industrial Pharmacy, vol. 1, no. 39, pp. 762769, 2013.

[18] J. P. Moeschwitzer and R. H. Mueller, "Factors influencing the release kinetics of drug nanocrystal-loaded pellet formulations," International Journal of Pharmaceutics, vol. 5, no. 450, pp. 278289, 2013.

[19] Z. Y. Zhang, S. H. Zhai, and J. H. Wang, "Preparation of cyhalothrin micro-emulsion formulation and its micro-droplet size," Agrochemicals, vol. 51, no. 5, pp. 351-357, 2012.

[20] Y. Liu, F.-S. Lu, T.-T. Chen, C.-L. Gao, H.-Y. Zhu, and H. Zhao, "Studies on the effect of different coherer counter-ions on forming rule and stability of cyhalothrin microemulsion," Chemical Journal of Chinese Universities, vol. 28, no. 5, pp. 909912, 2007.

[21] L. L. Chen, J. X. Chen, L. H. Chen et al., "Photodegradation of lambda-cyhalothrin in various solvents," Fine Chemical Intermediates, vol. 40, no. 6, pp. 16-19, 2010.

[22] SAC AQSIQ, "Determination method of suspensibility for pesticides," GB/T 14825-2006, Shenyang Research Institute of Chemical Industry, Shenyang, China, 2006.

[23] HB/T 2467.5-2003, Guidelines on Drafting Specifications of Pesticide Suspensions, Shenyang Research Institute of Chemical Industry, Shenyang, China, 2003.

[24] R. Mauludin and R. H. Müller, "Preparation and storage stability of rutin nanosuspensions," Journal of Pharmaceutical Investigation, vol. 43, no. 5, pp. 395-404, 2013.

[25] Y. C. Wang, Z. P. Liu, D. R. Zhang et al., "Development and in vitro evaluation of deacety mycoepoxydiene nanosuspension," Colloids and Surfaces B: Biointerfaces, vol. 83, no. 2, pp. 189-197, 2011.

[26] X.-J. Tang, Y.-H. Fu, Q.-H. Meng et al., "Evaluation of pluronic nanosuspensions loading a novel insoluble anticancer drug both in vitro and in vivo," International Journal of Pharmaceutics, vol. 456, no. 1, pp. 243-250, 2013.

[27] B.-D. Shen, C.-Y. Shen, X.-D. Yuan et al., "Development and characterization of an orodispersible film containing drug nanoparticles," European Journal of Pharmaceutics and Biopharmaceutics, vol. 85, no. 3, pp. 1348-1356, 2013.

[28] M. Talekar, J. Kendall, W. Denny, S. Jamieson, and S. Garg, "Development and evaluation of PIK75 nanosuspension, a phosphatidylinositol-3-kinase inhibitor," European Journal of Pharmaceutical Sciences, vol. 47, no. 5, pp. 824-833, 2012.

[29] Y. C. Wang, Y. Zheng, L. Zhang, Q. Wang, and D. Zhang, "Stability of nanosuspensions in drug delivery," Journal of Controlled Release, vol. 172, no. 3, pp. 1126-1141, 2013.

[30] W. Li, P. Quan, Y. Zhang et al., "Influence of drug physicochemical properties on absorption of water insoluble drug nanosuspensions," International Journal of Pharmaceutics, vol. 460, no. 1-2, pp. 13-23, 2014.

[31] J. Pardeike, D. M. Strohmeier, N. Schrödl et al., "Nanosuspensions as advanced printing ink for accurate dosing of poorly soluble drugs in personalized medicines," International Journal of Pharmaceutics, vol. 420, no. 1, pp. 93-100, 2011.
[32] W. Sun, S. R. Mao, Y. Shi, L. C. Li, and L. Fang, "Nanonization of itraconazole by high pressure homogenization: stabilizer optimization and effect of particle size on oral absorption," Journal of Pharmaceutical Sciences, vol. 100, no. 8, pp. 33653373, 2011.

[33] S. J. Prestrelski, T. Arakawa, and J. F. Carpenter, "Separation of freezing- and drying-induced denaturation of lyophilized proteins using stress-specific stabilization: II. Structural studies using infrared spectroscopy," Archives of Biochemistry and Biophysics, vol. 303, no. 2, pp. 465-473, 1993.

[34] S. J. Prestrelski, K. A. Pikal, and T. Arakawa, "Optimization of lyophilization conditions for recombinant human interleukin-2 by dried-state conformational analysis using Fourier-Transform infrared spectroscopy," Pharmaceutical Research, vol. 12, no. 9, pp. 1250-1259, 1995. 

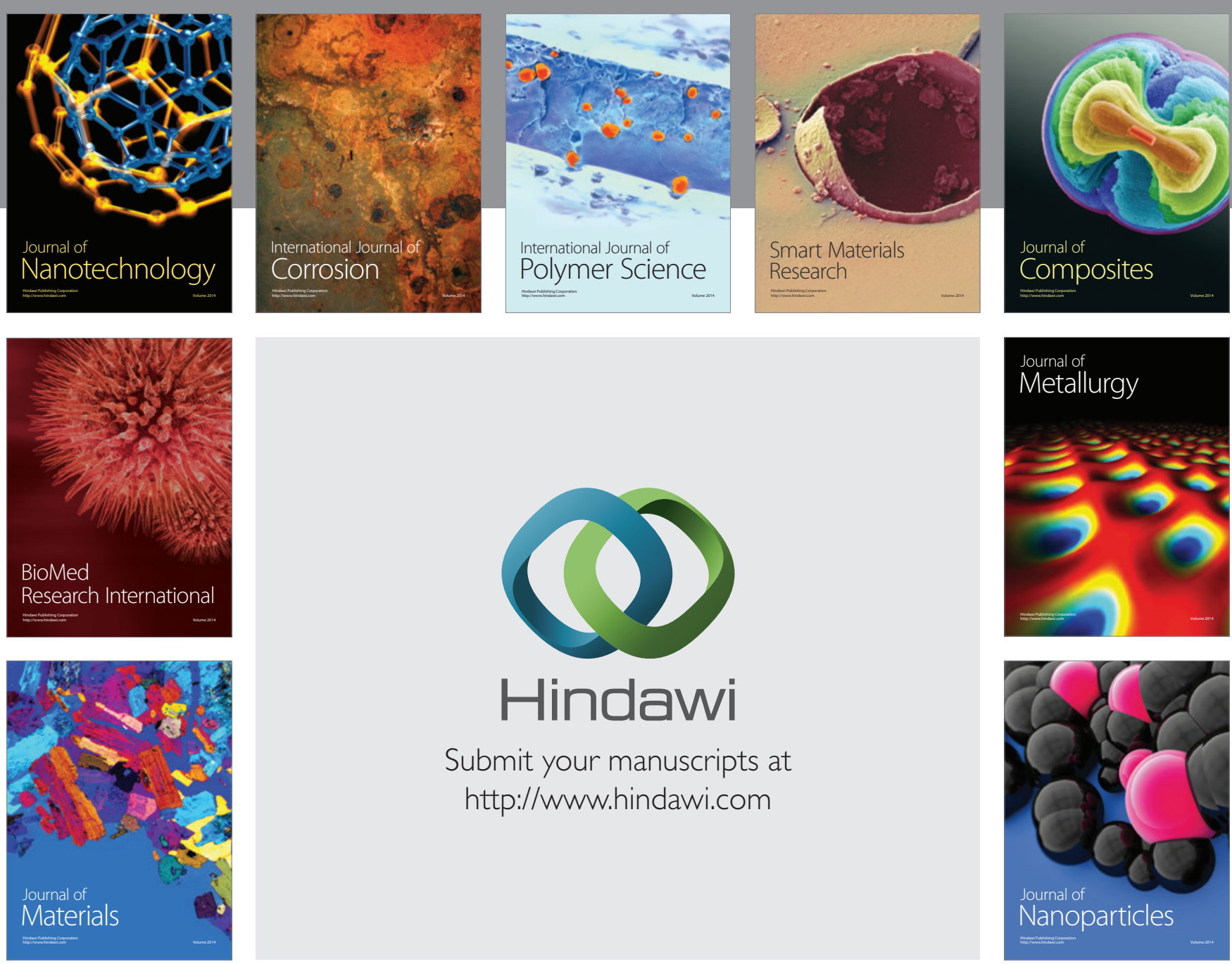

Submit your manuscripts at http://www.hindawi.com
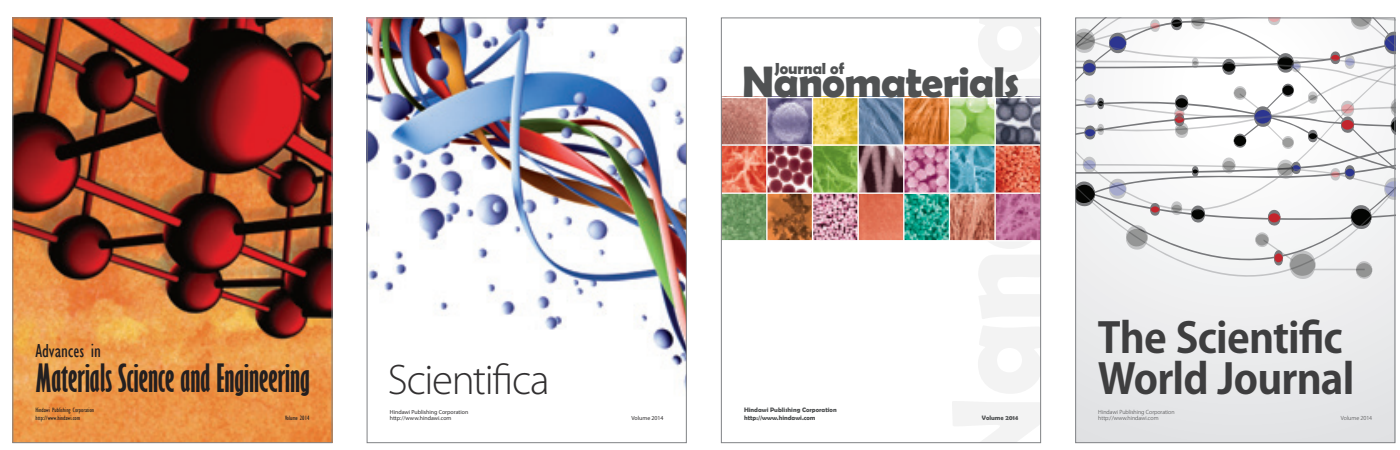

\section{The Scientific World Journal}
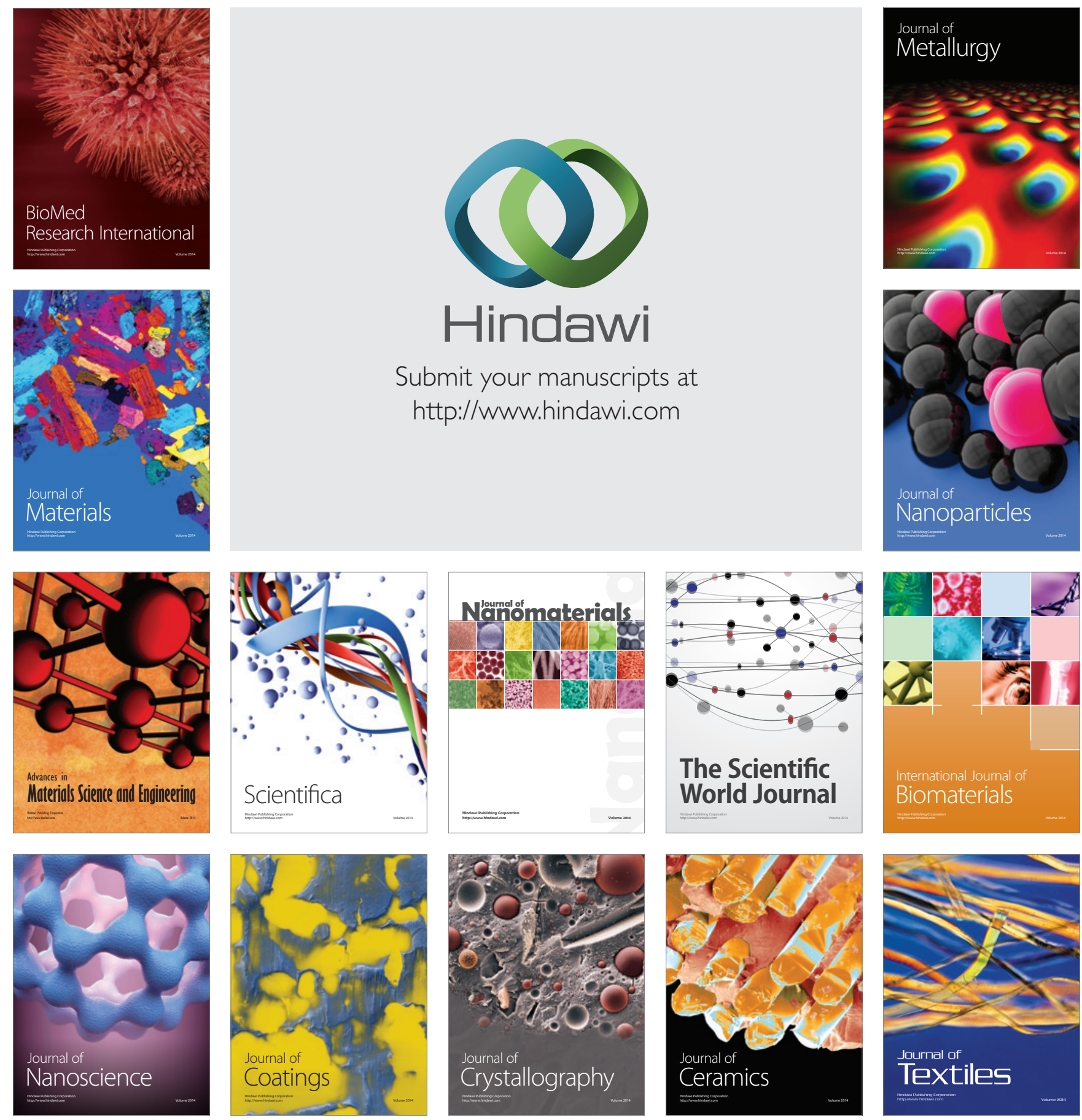